This is the accepted version of

Drennan V, Levenson R, Halter M, Tye C. Physician assistants in English general practice: a qualitative study of employers' viewpoints. J Health Serv Res Policy. 2011 Apr;16(2):75-80. doi: 10.1258/jhsrp.2010.010061. Epub 2011 Mar 9.

The published version can be found here http://hsr.sagepub.com/content/16/2/75.long

\title{
Physician assistants in English general practice: a qualitative study of employers' viewpoints.
}

\section{Abstract \\ Objective}

Effective use of staff is a major aim in all health care systems both to maximise their impact and to minimise costs. In England, a few general practitioners (GPs) have been recruiting physician assistants (PAs) to work in their practices, independent of any pilot schemes. Our objective was to study the motivation of GPs and practice managers who employed PAs and to understand the factors that sustained their employment.

\section{Methods}

A qualitative study using semi-structured interviews, analysed thematically, was carried out with 13 GPs and three practice managers from 15 general practices employing PAs in five areas of England.

\section{Results}

All practices were employing USA-trained PAs. Motivating factors for their employment included increasing the general practice capacity to manage patient demand within government targets for access, broaden the skill-mix in the practice team and financial considerations. The issues that needed to be taken into account in employing PAs included: the requirement for medical supervision; the PAs current lack of a regulatory framework and prescribing authority; and some patients' lack of familiarity with the concept of the PA. 


\section{Conclusions}

General practice employers view PAs as a positive addition to a mixed skill team for meeting patient demand within a practice's finances. There is a need to develop stronger governance and regulatory frameworks for this emerging profession. 


\section{Introduction}

Policy makers and managers in health care systems seek the most effective use of finite resources and in particular that of the workforce. Internationally, the human resource policies in health systems emphasise workforce flexibilities such as role re-design, blurring of professional role boundaries and expanding and training support workers. 'More staff working differently' through service re-design summed up the NHS workforce strategies in the face of shortages of all professional groups and a need to contain costs. ${ }^{2}$ One example of re-design has been the introduction of physician assistants (PAs) in UK primary health care.

The PA role in the UK is unregulated and the job title is used for two different types of staff. The first is a technician role working to the direction of junior hospital doctors. The second, and the focus of this paper, is the US model of PA, which has developed over the past 40 years in response to physician shortages in specialties such as primary care, difficulties in recruiting physicians to impoverished and rural areas, and minimising costs. ${ }^{3}$ Since the 1970 s demand for PAs has outstripped supply, particularly from solo primary care practices, ${ }^{4}$ with over 60,000 practising PAs in the USA. ${ }^{5}$ PAs graduate from a two year programme plus an internship year and are licensed to practice medicine in interdependent roles with physicians ${ }^{3}$. They undertake physical examinations, investigations, diagnosis, treatment, and prescribing within their scope of practice as agreed with their supervising physician. PA practice has been shown to be safe, equivalent to physicians for the types of case they attend and acceptable to patients. ${ }^{6-8}$ Their task productivity is between $50-80 \%$ of a physician. ${ }^{9}$ Cost savings to the organisation depend on the remuneration levels of individual PAs and physicians. ${ }^{10}$ 
The UK has been exploring the use of PAs, as have other countries. ${ }^{11,12}$ Two pilot projects evaluated USA trained Pas, employed for up to 24 months in six different clinical settings: out of hours, primary care, intermediate care, emergency medicine, acute receiving, and orthopaedics. The English pilot included 12 PAs, mainly employed in general practice ${ }^{13}$ and the Scottish pilot included 15 PAs of which 8 were employed in general practice or out of hours services. ${ }^{14}$ Both evaluations reported that the PAs were well accepted by patients, undertook work previously done by medical staff, needed to consult medical staff infrequently and exhibited greater diagnostic skills than extended roles in nursing, although there were caveats about the lack of UK regulation. ${ }^{13,14}$ A PA competency and curriculum framework has since been agreed by the Department of Health (England) and the Royal Colleges of Physicians and General Practitioners. ${ }^{15}$ Post graduate diploma courses commenced in four English Universities suggesting that there are potential employers of PAs. ${ }^{16}$ The widespread uptake of an innovation depends on the interaction between features of the innovation, the adopter(s), and the context. ${ }^{17,18}$ This study investigated this dynamic in relation to the uptake of PAs in primary care by addressing the perceptions and views of GPs who have chosen to employ PAs, outwith a pilot project, and their perception of the value and challenges in employing PAs.

\section{Methods}

Semi-structured interviews were administered by telephone in 2009. Telephone interviews were selected for efficiency with a dispersed sample ${ }^{19}$ of GPs for whom time and workload have been identified as barriers to participation in research. ${ }^{20}$ Employers of PAs were identified through internet searches using the term 'Physician Assistant', the UK Association of Physician Assistants (UKAPA) and snowball techniques, asking participants if they knew of any other general practice employers of PAs. This identified 22 PA employing general practices. 
Written information about the study and a request for an interview were sent to the senior partner at each of these practices. The letter was followed up by a telephone call during which oral consent to participate was requested and appointments made. The interview guide covered:

- Description of the work of the PA and how the PA works with GPs (e.g. pick up issues with GPs in the surgery at the time, debrief sessions, regular meeting with one GP)

- What informed your decision to employ/provide placements for a PA?

- What factors informed (or might inform) your decision to employ a PA rather than someone with a different professional background e.g. doctor or nurse practitioner?

- What are the advantages, benefits or any added value in employing a PA?

- What are the additional costs and issues that need to be taken into account in employing a PA?

Detailed notes were taken during the interview and pseudonymised using a study identity number. The notes were read and analysed by each of the authors, independently of each other, in an inductive process. A group discussion was held in which each author's thematic interpretation was presented. Any difference in opinion was discussed and resolved as a group, resulting in a set of agreed themes. A draft report was circulated to participants who were offered the opportunity to remove any part or all of their data from the study.

\section{Results}

\section{Participants}

Fifteen GPs were recruited to the study. In three practices the practice manager also volunteered to participate. In two of these practices the GP was not then able to participate due to time constraints. Telephone interviews were therefore conducted with 13 GPs and three practice managers in 15 general practices employing PAs in total. Participants came from five areas of England: East of England, London, South Central, South West, and West Midlands. The fifteen practices ranged from a large team of partners and salaried GPs with up to three PAs at any one time through to a single- 
handed GP practice working with two PAs. All were employing USA trained PAs. One participant was also employing a recent graduate of a UK programme. Most participants had been employing USA trained PAs for over two years and one for just under a year.

Four main issues were addressed by participants: motivation for employing PAs; the work of the PAs in the practice team; the benefits and challenges of employing PAs.

\section{Motivation for employing PAs in general practice}

Many reasons were given for the initial decision to employ a PA. These included meeting government access time targets, increased patient demand, recruitment problems, and cost effectiveness:

'Basically, it was about access, appointments, and affordability'. Participant 3

One of the most frequently reported motives for starting to use PAs was the need to manage patient demand, particularly having to see patients on the same day as required by the Department of Health. ${ }^{22}, 23$ Linked to this was a perception that the nature and volume of work in primary care had changed; an increasing volume of minor illness consultations that required a different skill mix in general practice. Some participants thought that introducing PAs might allow GPs to apply their skills to the more complex patients.

'It is not only a question of cost. It can be mind-numbing for an over qualified person to deal with simple problems. It is appropriate to have people with a range and levels of expertise'.

\section{Participant 1}

A further factor was the difficulty experienced in recruiting doctors and nurse practitioners, particularly in practices in deprived urban areas and rural areas. In some instances the Primary Care Trusts had actively supported recruitment of USA trained PAs. 
For several participants, the cost of a PA compared to a doctor was the most persuasive factor though there were mixed views on comparative costs. Some interviewees spoke of the relatively low cost of PAs compared to GPs for similar levels of work, while others considered that PAs were not necessarily a cheaper option, due to lower productivity and the need for medical supervision. This difference in views was reflected in their on-going motivation to employ PAs. While some participants were convinced of a place for PAs in their practice teams and promoted the role to other GPs, others considered that each decision on staffing should be taken in the light of the current context e.g. the demands of the general medical services contract, the patient workload and the skill set of the current staff.

\section{The work of the PAs in general practice}

Participants described an incremental induction process to both accustom new PAs to English primary care and also assure GPs of their competence and safety. This involved the GP observing a PA's consultations with patients with minor conditions before expanding the types of patients seen by PAs. All of the practices had systems in place for both regular supervision and the provision of immediate medical advice to PAs if it was needed.

'They can speak to the GPs at any time if there is a need. I am the officially designated supervising physician. The PAs get regular supervision, especially at the start, both for their benefit and for us to be satisfied that they know what they are doing. Then, once their feet are under the table it's a monthly review meeting specific for the PA, and they take part in a weekly clinical meeting with all the doctors too' Participant 12

Irrespective of the motivation, PAs were deployed to triage patients and/or see same-day appointments. 
'The work of the PA is not filtered. She does a lot of the same day, emergency work. She does some follow-ups of her own choosing. She organises her own investigations and referrals'

\section{Participant 16}

Occasionally PAs carried out reviews and management of people with long-term conditions but this was mainly the domain of the practice nurses. Some PAs occasionally undertook home visits to patients though this was limited by their lack of prescribing authority.

\section{The benefits of employing PAs}

In addition to the reasons for employing PAs, participants described other benefits. PAs were perceived as able to do a high volume of work at the same time as requiring low levels of supervision. Participants valued PAs' ability to work within a medical framework, to manage uncertainty and to make decisions. GPs contrasted this to employing nurses who were seen as either less productive or in need of more supervision.

I have had experience of nurse practitioners and, I don't mean this pejoratively, they think like nurses, and work to protocols and systems. PAs have had a medical training and they have a style of diagnostic working and can think on their feet.' Participant 16

PAs were also seen as acceptable to patients and this was illustrated by some participants citing examples of patients specifically asking to see the PA, although the reasons for this were unclear. A separate issue was patients' understanding of what a PA was. Practices had developed different approaches to informing patients that they might be seen by a PA and getting their consent to this. However there remained some doubts as to patients' understanding of exactly what a PA was and that they were not a doctor:

'Patients have no problems with seeing a PA. They have no idea what a PA is. They talk about seeing "the American doctor", although we don't call her doctor'. Participant 15 


\section{The challenges and costs in employing PAs}

All participants reported that the advantages of employing a PA outweighed or at least balanced the costs and challenges. They were consistent in pointing to the same challenges: in particular, the lack of prescribing authority. GPs had devised a range of methods for overcoming this, such as waiting for a GP to be free to sign the prescription:

'Signing prescriptions - they leave it out for us to sign, and once we have ensured that we are comfortable with how they prescribe, that's what we do. Nurse practitioners have a protocol, but we don't require that as much from the PAs as they have more training for the role. We do look at all the prescriptions, but we don't formally check up on each one. But we do restrict their prescribing of controlled drugs - they would have to come and talk to us first about that' Participant 10

Other aspects of governance were also raised. The GPs were well aware of indemnity and liability issues and had secured appropriate insurance for the PAs' work. The question of risk and patient safety was taken seriously: one GP reported that US trained PAs were particularly litigationconscious. Nevertheless, several interviewees indicated that more attention was needed from statutory authorities on this issue, including the need for PAs to become a regulated profession in the UK and an enhancement of public awareness of PAs.

All participants raised the issue of the cost to the practice of employing a PA. While the salary of a PA was less than that of a doctor, additional costs arose. One was GP time supervising, although some people felt this was no greater for PAs than for any other staff. Some of the initial investment of GP time was thought by some to relate to the need for US-trained PAs to acclimatise to UK medical practices such as different thresholds for referral to secondary care. 
'She (the PA) was not good at limiting her referrals - they have things on tap in the States and their hospitals encourage referrals. She would also try and encourage patients to come back too often - again this is what they do in the States' Participant 9.

The other issue focused on PAs' productivity. Related to this was the relative advantages and disadvantages of UK or USA trained PAs. US trained PAs meant that the employer could choose from very experienced applicants, although there was the attendant issue of the different health care culture. However, while UK trained PAs would be culturally acclimatised to UK health care practices, they would not have so much experience. Some GPs mentioned that there were issues around how best to retain USA trained PAs who sometimes didn't stay long. Continuing professional development was cited as a cost, with few opportunities currently available in the UK. Several participants reported funding their PAs to return to the USA for professional development necessary for their registration. These costs were described as acceptable by employers.

The issue of acceptability of PAs to other professionals in the practice team, mostly nurses, was also raised. Most respondents noted that initially there was some form of 'wariness', bordering on resistance and hostility. Some participants reported that they had tried to address this as part of the process of employing a PA. Occasionally other doctors were reported to be mistrustful of the employment of PAs on the grounds that they might be in competition for jobs.

'We have got an F2 doctor who thinks he will be out of a job as PAs are cheaper to employ. But I say to him: there's no-one with a 2 year training who can replace a fully trained doctor' Participant 8

Examples were given of doctors in secondary care unwilling to accept referrals signed by PAs. The GPs addressed this in various ways, such as confirming to medical teams they endorsed these 
referrals. In some instances the GPs reported switching their referral patterns to other consultants and hospitals.

\section{Discussion}

Only a small number of GPs in England were employing PAs in 2009. The motivation for employing them was in response to meeting general practice contract requirements as efficiently and effectively as possible, within local workforce constraints. Participants viewed PAs as a beneficial, safe workforce solution although the lack of a regulatory framework and prescribing authority were seen as hindering their full potential. There were mixed views as to the cost effectiveness of employing PAs .

This is a small qualitative study from only the employers' perspective and as such has limitations. It does, however, include general practice employers from different parts of urban and rural England, in different sized practices and with different practice staff configurations. As such it illuminates an emerging aspect of primary care staffing.

There are 10,000 general practices in the UK, characterized by entrepreneurial developments ${ }^{23}$ ;some as a result of individual enthusiasm, ${ }^{24}$ others in response to incentives and labour market fluxes ${ }^{25,26}$. The flexibilities surrounding the 2004 general medical services contract have been used to create new organisational forms, deploy general practice nurses differently ${ }^{27}$, and employ increasing numbers of salaried doctors. ${ }^{28}$ Evidence suggests that appropriately trained nurses provide equivalent care to doctors for patients presenting with minor illness ${ }^{29}$ and that nurse practitioners could undertake at least $25 \%$ of the work currently undertaken by GPs ${ }^{30}$ The recruitment and retention of experienced nurses into UK primary care has proved challenging particularly in areas such as the South East ${ }^{31}$ 
Against this backdrop, the participants in this study used criteria such as those suggested by Greenhalgh et $\mathrm{al}^{17}$ to judge the advantages of adopting this staffing innovation. They all reported benefits of employing a mix of differently skilled staff, including medically oriented 'advanced' or 'mid-level' practitioners. Their positive approach reflects some UK general practitioner commentaries on the PA role ${ }^{32}$ but these contrast with less supportive views expressed elsewhere. ${ }^{33}$

The challenges reported of working with a professional group that is not regulated echo those of the earlier pilot studies ${ }^{13,14}$ and reflect ongoing debates in the UK. The variety of views expressed on the cost benefit of PAs (experienced and inexperienced) relative to doctors or nurse practitioners in general practice suggest that more detailed investigation is required. A funding application has been successful to investigate the contribution of PAs to primary care in England from multiple stakeholder perspectives, including patients, with more detailed economic analysis and commences in $2010^{34}$

While many participants were convinced of the future place of the PA, others argued that changes in the local and national context for their practice would shape their decisions about employing PAs, just as they did for decisions about any staff. This context is changing both in terms of supply and demand for health care professionals. Medical schools in the UK have expanded in the last decade and in the NHS Employers' view, an oversupply of doctors is seen as beneficial. ${ }^{35}$ Newly qualified general practitioners are currently reporting difficulties in finding positions ${ }^{36}$ as the health service seeks greater productivity at lower cost and service re-design. ${ }^{37}$ It remains to be seen how these forces influence the innovation of PA roles in primary care in the UK. 


\section{Acknowledgements}

The authors thank the study participants for their time and willingness to be interviewed, and the

UKAPA for contacting their members on our behalf. 


\section{References}

1. Dal Poz M, Quain E, O'Neil M, McCaffery J, Elzinga G, Martineau T. Addressing the health workforce crisis: towards a common approach. Human Resources for Health 2006; 4: 21.

2. Department of Health. Human Resources in the NHS Plan: More Staff Working Differently. London: Department of Health, 2002.

3. Mittman DE, Cawley JF, Fenn WH. Physician assistants in the United States. BMJ 2002; 325:485-7

4. Scheffler RM, Gillings DB. Survey approach to estimating demand for physician assistants. Soc Sci Med 1982; 16: 1039-47.

5. National Centre for Health Statistics. Health, United States 2008. Washington: National Centre for Health Statistics, 2009.

6. Ruby EB, Davidson LJ, Daly B et al: Care activities and outcomes of patients cared for by acute care nurse practitioners, physician assistants, and resident physicians: a comparison. $A m$ J Crit Care 1998; 7:267-81

7. Cawley JF, Rohrs FC, Hooker RS: Physician assistants and malpractice risk: findings from the national practitioner data bank. Fed Bull 1999; 85:242-6

8. Hooker RS, Cipher DJ, Sekscenski E. Patient satisfaction with physician assistant, nurse practitioner, and physician care: a national survey of Medicare beneficiaries. J Clin Outcomes Manag 2005; 12:88-92

9. Larson E, Hart LG, Ballweg R: National Estimates of Physician Assistant Productivity. $J$ Allied Health 2001; 30:146-152

10. Roblin DW, Howard DH, Becker ER, Adams EK, Roberts MH. Use of midlevel practitioners to achieve labor cost savings in the primary care practice of an MCO. Health Serv Res 2004; 39:607-625

11. O'Connor TM, Hooker RS: Extending rural and remote medicine with a new type of health worker: Physician assistants. Aust J Rural Health 2007; 15:346-351

12. McCabe D. The next wave: "physician extenders"? CMAJ 2007; 177:447

13. Woodin J, McLeod H, McManus R. Evaluation of US-trained physician assistants working in the NHS in England: the introduction of US-trained physician assistants to primary care and accident and emergency departments in Sandwell and Birmingham: Final report. Health Services Management Centre, University of Birmingham; 2005.

(http://www.hsmc.bham.ac.uk/publications/Archive.shtml\#2005).

14. Farmer J, Currie M, West C, Hyman J, Arnott N. Evaluation Of Physician Assistants To NHS Scotland: Final Report. Inverness: UHI Millennium Institute, 2009.

(http://www.nes.scot.nhs.uk/documents/publications)

15. Department of Health. The Competence and Curriculum Framework for the Physician Assistant. London: Department of Health, 2006.

16. Rogers EM. Diffusion of Innovations. ( $5^{\text {th }}$ edition) New York: Free Press, 2003.

17. Greenhalgh T, Robert G, Macfarlane F, Bate P, Kyriakidou O. Diffusion of innovations in service organizations: systematic review and recommendations. Milbank $Q$ 2004; 82:581-629

18. May C, Finch T, Mair F, et al. Understanding the implementation of complex interventions in health care: the normalization process model. BMC Health Services Research 2007; 7:148

19. Sturges JE, Hanrahan KJ. Comparing Telephone and Face-to-Face Qualitative Interviewing: a Research Note.Qualitative Research 2004;4: 107-118.

20. Hummers-Pradier E, Scheidt-Nave C, Martin H, Heinemann S, Kochen MM and Himmel W: Simply no time? Barriers to GPs' participation in primary health care research. Family

Practice 2008; 25:105-12. 
21. Department of Health. Priorities and Planning Framework (2003 - 2006). London: Department of Health, 2003.

22. Department of Health: The NHS in England: the Operating Framework for 2007/2008. London: Department of Health, 2006.

23. Huntingdon J .From Invention to Innovation pp 211-220 in Meads G (ed) Future Options for General Practice. Oxford : Radcliffe Medical Press, 2004.

24. Jones R, Rosen R, Tomlin Z, Cavanagh MR, Oxley D. General practitioners with special interests: evolution and evaluation. J Health Serv Res Policy 2006; 11:106-9.

25. Illife S. From General practice to Primary Care: The Industrialization of Family Medicine. Oxford: Oxford University Press, 2008.

26. Exworthy M, Wilkinson EK, McColl A, et al. The role of performance indicators in changing the autonomy of the general practice profession in the UK. Soc Sci Med 2003;56:1493-504

27. Working in Partnership Project [WiPP]. Minor Illness Management Case Studies undated accessed last on 15/1/10 at http://www.pagb.co.uk/information/minorillnessmanagement.html

28. Royal College of General Practice. A Profile of UK General Practitioners. London : Royal College of General Practice, 2006.

29. Laurant M, Reeves D, Hermens R, Braspenning J, Grol R, Sibbald B. Substitution of doctors by nurses in primary care. Cochrane Database Syst Rev 2005; 18:CD001271.

30. Wanless D. HM Treasury. Securing good health for the whole population: Final report. London: HM Treasury, 2004.

31. Meldrum H, Malone B . Letter to General Practitioners regarding practice nurses and the Agenda for Change. London: General Practitioners Committee of the British Medical Association, 2006.

32. Lord J. Future of primary healthcare education: current problems and potential solutions. Postgraduate Medical Journal 2003; 79:553-560

33. Van Der Weyden MB. Doctor displacement: a political agenda or health care imperative? Med J Aust 2008; 189:608-9

34. NIHR SDO PC245 09-1801-1006. Investigating the contribution of physician assistants (PAs) to primary care in England. Drennan V, Halter M, De Lusignan S, Gage H , Gabe J and Brearley S.

35. NHS Employers. Briefing Paper 52. Medical Training and Careers the Employers Vision. London: 2008.

36. Hutt P. What has happened to general practice? BMJ Careers Online 13 March 2009 http://careers.bmj.com/careers/advice/view-article.html?id=20000088

37. Appleby J, Crawford R, Emmerson C. How cold will it be? Prospects for NHS funding: 2011-17. London: Kings Fund 